\title{
A note on the motion of a spherical particle in a general quadratic flow of a second-order fluid
}

\author{
By P. C.-H. CHAN AND L. G. LEAL \\ Department of Chemical Engineering, California Institute of Technology, Pasadena
}

(Received 12 July 1976 and in revised form 10 December 1976)

The migration of a rigid sphere in a two-dimensional unidirectional shear flow of a second-order fluid was considered by Ho \& Leal (1976). It was found that the sphere would migrate in the direction of decreasing absolute shear rate. The present paper extends the previous results to a general quadratic flow, and also considers the case of a spherical drop.

\section{Introduction}

Brenner (1964) studied the Stokes resistance of an arbitrary rigid particle in an arbitrary field of flow. He showed that both the force and the torque relative to any given point $O$ of the body can depend only linearly on certain vectorial or tensorial parameters that characterize the undisturbed bulk velocity distribution. In a general linear flow, these parameters are the translational velocity relative to that of the particle, the vorticity tensor as seen in a frame of reference which rotates with the particle, and the rate-of-strain tensor. Calculation of the force and torque from these quantities, for an arbitrary body, then reduces to the determination of six tensor coefficients which depend only on the shape of the body. Symmetry conditions for these tensors were discussed by Hinch (1972).

Application of these general ideas to the motion of an arbitrary rigid particle in a non-Newtonian fluid was first reported by Brunn $(1976,1977)$, who used an incompressible second-order fluid in a perturbation expansion about the Newtonian limit. As a consequence, the non-Newtonian contributions to the force and torque were found to depend only on quadratic combinations of the flow parameters. However, Brunn considered only a linear bulk velocity field and concluded that no migration would occur for neutrally buoyant rigid particles. In contrast, Ho \& Leal (1976) recently reported detailed calculations for the motion of a rigid sphere in a two-dimensional, quadratic, unidirectional shear flow of a second-order fluid. In this case, it was found that the sphere would migrate in the direction of decreasing absolute shear rate.

The present paper extends the results of Ho \& Leal (1976) to a general quadratic flow of a second-order fluid. In $\$ 2$ we outline the formulation of the problem. In $\$ 3.1$ general expressions are obtained for the translation and rotation of a rigid sphere, and numerical values for the geometry-dependent coefficients which appear are determined by comparison with the detailed calculation for the specific flow of Ho \& Leal (1976). Finally, in §3.2 we obtain analogous results for a spherical drop. As will be obvious later on, index notation is to be preferred over tensor notation for our analysis and will be used throughout this paper. 


\section{Formulation of the problem}

We consider a rigid particle suspended in a second-order fluid which is undergoing some general quadratic motion. To non-dimensionalize, we define a characteristic (particle) length $a$, a characteristic velocity $G a$ and a characteristic pressure $\mu_{0} G$, where $G$ is an average shear rate for the bulk flow and $\mu_{0}$ is the zero-shear viscosity. We adopt a non-rotating co-ordinate system which has its origin fixed at the centre of rotation of the particle, and thus translates with the particle velocity $\left(U_{s}\right)_{i}$ relative to a fixed laboratory reference frame. The position vector of a point $R$ in this translating frame is denoted by $x_{i}$, whereas the position vectors for $R$ and for the centre of rotation measured with respect to the fixed frame are $x_{i}^{\prime}$ and $\left(x_{0}(t)\right)_{i}$, respectively. Thus

$$
x_{i}=x_{i}^{\prime}-\left(x_{0}(t)\right)_{i} \text {. }
$$

The complete dimensionless velocity and pressure distributions, including the disturbance motion induced by the particle, will be denoted by $\left(U_{i}, P\right)$. The equations of motion, with inertia effects neglected, are then

$$
\partial S_{i j} / \partial x_{j}=0, \quad \partial U_{i} / \partial x_{i}=0
$$

where

$$
S_{i j}=-P \delta_{i j}+D_{(1) i j}+\lambda D_{(1) i k} D_{(1) k j}+\lambda \epsilon_{1} D_{(2) i j} .
$$

$D_{(1) i j}$ and $D_{(2) i j}$ are Rivlin-Ericksen tensors given by

$$
\begin{gathered}
D_{(1) i j}=\partial U_{i} / \partial x_{j}+\partial U_{j} / \partial x_{i} \\
D_{(2) i j}=\partial D_{(1) i j} / \partial t+U_{k} D_{(1) i j, k}+D_{(1) i k} U_{k, j}+D_{(1) k j} U_{k, i}
\end{gathered}
$$

while $\lambda$ and $\epsilon_{1}$ are material parameters of the fluid. From a macroscopic point of view, $\lambda$ is often defined as the ratio of a normal-stress function to the viscosity in the limit of zero shear; physically, it may be interpreted as the ratio of the intrinsic relaxation time scale of the fluid to the convective time scale of the flow problem (Caswell \& Schwarz 1962). We assume here that the flow is slow (nearly Newtonian) compared with the intrinsic relaxation time (i.e. $\lambda \ll 1$ ) but that non-Newtonian effects are still more important than inertial effects (i.e. $R e \ll \lambda$ ). Similarly, the undisturbed bulk velocity and pressure fields $\left(V_{i}, Q\right)$ may be assumed to satisfy the equations

$$
\partial T_{i j} / \partial x_{j}=0, \quad \partial V_{i} / \partial x_{i}=0,
$$

where

$$
T_{i j}=-Q \delta_{i j}+E_{(1) i j}+\lambda E_{(1) i k} E_{(1) k j}+\lambda \epsilon_{1} E_{(2) i j}
$$

with $E_{(n) i j}$ defined analogously to $D_{(n) i j}$, but using $V_{i}$.

We are interested primarily in the $O(\lambda)$ contribution to the translational velocity of the particle. For a unidirectional flow, this specifies the lateral migration as well as any $O(\lambda)$ contributions to motion in the direction of the undisturbed bulk flow. Ho \& Leal (1976) showed rigorously, in the case of a sphere in a two-dimensional unidirectional flow, that the non-Newtonian migration is a 'near-field' effect caused by the disturbance velocity field in the vicinity of the sphere, provided of course that the sphere is small relative to the characteristic dimension of the flow $d$ (i.e. $\zeta=a / d \ll 1$ ). In particular, the fluid can effectively be considered as unbounded, with no direct effect of the bounding walls other than their role in determining the undisturbed velocity profile. Any corrections to the infinite-domain disturbance flow to account for the 
presence of boundaries give only higher-order corrections in $\zeta$ for the migration velocity. A rigorous proof that the non-Newtonian contributions to particle motion at $O(\lambda)$ are similarly dominated by 'near-field' effects for a general quadratic flow has not been constructed. $\dagger$ Nevertheless, we believe this result to hold true and shall therefore assume that the presence of any walls may be neglected. Hence we require that the complete velocity field $U_{i}$ reduces to the undisturbed form at large distances from the particle, i.e.

$$
U_{i} \rightarrow V_{i} \text { as } r=\left(x_{j} x_{j}\right)^{\frac{1}{2}} \rightarrow \infty .
$$

On the surface of the particle, $U_{i}$ satisfies

$$
U_{i}=-\epsilon_{i j k}\left(\omega_{s}\right)_{k} x_{j}
$$

The undisturbed velocity relative to the laboratory-fixed reference frame, which we denote by $V_{i}^{\prime}$, is assumed to be steady in time and representable, at any instant, by the general quadratic form

$$
\dot{V}_{i}^{\prime}=\alpha_{i}^{\prime}+\beta_{i j}^{\prime} x_{j}^{\prime}+\gamma_{i j k}^{\prime} x_{k}^{\prime} x_{j}^{\prime},
$$

where $\alpha_{i}^{\prime}, \beta_{i j}^{\prime}$ and $\gamma_{i j k}^{\prime}$ are constant tensors. Thus, in the translating frame which we have adopted,

where

$$
\left.\begin{array}{c}
V_{i}=\alpha_{i}+\beta_{i j} x_{j}+\gamma_{i j k} x_{k} x_{j}-\left(U_{s}\right)_{i}, \\
\alpha_{i}=\alpha_{i}^{\prime}+\beta_{i j}^{\prime}\left(x_{0}\right)_{j}+\gamma_{i j k}^{\prime}\left(x_{0}\right)_{k}\left(x_{0}\right)_{j}, \\
\beta_{i j}=\beta_{i j}^{\prime}+2 \gamma_{i j k}^{\prime}\left(x_{0}\right)_{k}, \quad \gamma_{i j k}=\gamma_{i j k}^{\prime} .
\end{array}\right\}
$$

The coefficient $\gamma_{i j k}$ remains constant in time, but $\alpha_{i}$ and $\beta_{i j}$ are time dependent, as a result of the motion of the reference frame. It follows easily from (2.11) that

$$
\partial \alpha_{i} / \partial t=\beta_{i j}\left(U_{s}\right)_{j}, \quad \partial \beta_{i j} / \partial t=2 \gamma_{i j k}\left(U_{s}\right)_{k} .
$$

In deriving (2.11) and $(2.12 a, b)$, we have made use of the obvious symmetry condition

$$
\gamma_{i j k}=\gamma_{i k j}
$$

It is also apparent from $(2.5 b)$ that $\beta_{i j}$ and $\gamma_{i j k}$ satisfy the additional constraints

$$
\beta_{i i}=0, \quad \gamma_{i k i}=\gamma_{i i k}=0 .
$$

In this paper, tensors which give zero when contracted along any two indices will be termed completely irreducible.

Both $\beta_{i j}$ and $\gamma_{i j k}$ have to be decomposed into their respective irreducible components in order to be applied conveniently in a general expression for the force or torque. This decomposition was illustrated by Coope, Snider \& McCourt (1965). Its main advantage for present purposes is that all the components of $\beta_{i j}$ and $\gamma_{i j k}$ are made to be completely irreducible and symmetric, and much simplification is then possible in the analysis. Besides, each component then has its own physical significance. The decomposition of $\beta_{i j}$ is well known:

Consequently

$$
\beta_{i j}=\frac{1}{2}\left(\beta_{i j}+\beta_{j i}\right)+\frac{1}{2}\left(\beta_{i j}-\beta_{j i}\right) .
$$

where

$$
\beta_{i j} x_{j}=e_{i j} x_{j}-\epsilon_{i j k} \Omega_{k} x_{j},
$$

$\dagger$ And, indeed, would be extremely difficult in this general problem. 
The decomposition of $\gamma_{i j k}$ is more complicated:

$$
\begin{aligned}
\gamma_{i j k}= & {\left[\frac{1}{6}\left(\gamma_{i j k}+\gamma_{i k j}+\gamma_{k i j}+\gamma_{k j i}+\gamma_{j i k}+\gamma_{j k i}\right)\right.} \\
& \left.-\frac{1}{15}\left(\gamma_{i m m} \delta_{j k}+\gamma_{j m m} \delta_{i k}+\gamma_{k m m} \delta_{i j}\right)\right]+\frac{1}{3}\left[\epsilon_{i j l}\left(-\epsilon_{l m n} \gamma_{n m k}-\epsilon_{k m n} \gamma_{n m l}\right)\right. \\
& \left.+\left(-\frac{1}{2} \epsilon_{i m n} \gamma_{n m l}-\frac{1}{2} \epsilon_{l m n} \gamma_{n m i}\right) \epsilon_{l j k}\right]+\frac{1}{10}\left[-\gamma_{k m m} \delta_{i j}-\gamma_{j m m} \delta_{i k}+4 \gamma_{i m m} \delta_{j k}\right] .
\end{aligned}
$$

Consequently

$$
\begin{aligned}
\gamma_{i j k} x_{k} x_{j}= & \psi_{i j k} x_{k} x_{j}-\frac{1}{3}\left(\epsilon_{i j l} \delta_{m k}+\frac{1}{2} \delta_{i m} \epsilon_{l j k}\right) \theta_{l m} x_{k} x_{j} \\
& +\frac{1}{1}\left(-\delta_{k l} \delta_{i j}-\delta_{j l} \delta_{i k}+4 \delta_{i l} \delta_{j k}\right) \tau_{l} x_{k} x_{j},
\end{aligned}
$$

where

$$
\left.\begin{array}{rl}
\psi_{i j k}= & \frac{1}{6}\left(\gamma_{i j k}+\gamma_{i k j}+\gamma_{k i j}+\gamma_{k j i}+\gamma_{j i k}+\gamma_{j k i}\right) \\
& -\frac{1}{15}\left(\gamma_{i m m} \delta_{j k}+\gamma_{j m m} \delta_{i k}+\gamma_{k m m} \delta_{i j}\right), \\
\theta_{i j}= & \epsilon_{i m n} \gamma_{n m j}+\epsilon_{j m n} \gamma_{n m i}, \quad \tau_{i}=\gamma_{i m m} .
\end{array}\right\}
$$

Once again, we note that $e_{i j}, \psi_{i j k}$ and $\theta_{i j}$ are all completely irreducible and symmetric. It is also important to observe that both

$$
\left(\epsilon_{i j l} \delta_{m k}+\frac{1}{2} \delta_{i m} \epsilon_{l j k}\right) \theta_{l m} \text { and }\left(-\delta_{k l} \delta_{i j}-\delta_{j l} \delta_{i k}+4 \delta_{i l} \delta_{j k}\right) \tau_{l}
$$

in (2.19) are symmetric in $j, k$ (the former condition is by no means obvious from a casual inspection) and therefore our decomposition is consistent with (2.13).

Intuitively, it is appealing to interpret $\theta_{i j}$ as the vorticity gradient, and to assume that $\psi_{i j k}$ and $\tau_{i}$ specify the rate-of-strain gradient. To show that this intuitive 'guess' is indeed correct, we may re-express $V_{i}$ as

$$
\begin{aligned}
V_{i}=\alpha_{i}+e_{i j} x_{j}-\epsilon_{i j k} \Omega_{k} x_{j}+\psi_{i j k} & x_{k} x_{j}-\frac{1}{3}\left(\epsilon_{i j l} \delta_{m k}+\frac{1}{2} \delta_{i m} \epsilon_{l j k}\right) \theta_{l m} x_{k} x_{j} \\
& +\frac{1}{10}\left(-\delta_{k l} \delta_{i j}-\delta_{j l} \delta_{i k}+4 \delta_{i l} \delta_{j k}\right) \tau_{l} x_{k} x_{j}-\left(U_{s}\right)_{i}
\end{aligned}
$$

and follow the familiar argument based upon the rate of change of the length of a material line element (Fredrickson 1964) to get (using the results of last paragraph)

$$
d V_{i} \frac{d x_{i}}{d s}=e_{i j} d x_{j} \frac{d x_{i}}{d s}+2 \psi_{i j k} x_{k} d x_{j} \frac{d x_{i}}{d s}+\frac{1}{5}\left(-\delta_{k l} \delta_{i j}-\delta_{j l} \delta_{i k}+4 \delta_{i l} \delta_{j k}\right) \tau_{l} x_{k} d x_{j} \frac{d x_{i}}{d s}
$$

Finally we observe that

$$
d V_{i}\left(d x_{i} / d s\right)=\mathfrak{b}(d s) / \mathfrak{b} t,
$$

where $\delta / b t$ is a convected time derivative expressed in terms of a fixed co-ordinate system, and therefore

$$
\delta(d s)^{2} / \mathrm{b} t=2\left[e_{i j}+2 \psi_{i j k} x_{k}+\frac{1}{5}\left(-\delta_{k l} \delta_{i j}-\delta_{j l} \delta_{i k}+4 \delta_{i l} \delta_{j k}\right) \tau_{l} x_{k}\right] d x_{j} d x_{i}
$$

The argument for the vorticity is also well known and follows in a similar manner by consideration of the rate of rotation of a line element. For the sake of brevity, we shall omit the details here. As expected, the rotation depends only on $\Omega_{i}$ and $\theta_{i j}$.

\section{The migration velocity}

We now attempt to obtain general expressions for the force and torque on the particle. To this end, we first note that the solution of (2.2), obtained by a straightforward perturbation expansion, shows that the $O(1)$ Newtonian contribution is 
linear in the flow parameters $\left(\alpha_{i}-\left(U_{s}\right)_{i}\right), e_{i j}, \Omega_{i}, \psi_{i j k}, \theta_{i j}$ and $\tau_{i}$ and also the boundary value $\left(\omega_{s}\right)_{i}$. On the other hand, the $O(\lambda)$ non-Newtonian contribution consists of quadratic combinations of these seven parameters, from the time-independent terms in the non-Newtonian part of (2.3), and also additional linear terms arising from their time derivatives, due to particle translation. By this formulation, any contributions from the rate of change of orientation (i.e. rotation) of the particlo are included automatically. Hence, if we now use the superscript $(n)$ to denote contributions at $O\left(\lambda^{n}\right)$, the expressions for the force and torque are

$$
\begin{aligned}
F_{i}= & F_{i}^{(0)}+\lambda F_{i}^{(1)} \\
= & \left\{A_{i j}^{1}\left[\hat{\alpha}_{j}-\lambda\left(U_{s}^{(1)}\right)_{j}\right]+{ }^{t} A_{i j k}^{1} e_{k j}+{ }^{t} B_{i j}^{1} \Omega_{j}+{ }^{t} A_{i j k l}^{1} \psi_{l k j}+{ }^{t} B_{i j k}^{1} \theta_{k j}\right. \\
& \left.+{ }^{t} A_{i j}^{2} \tau_{j}+{ }^{t} B_{i j}^{2}\left[\left(\omega_{s}^{(0)}\right)_{j}+\lambda\left(\omega_{s}^{(1)}\right)_{j}\right]\right\} \\
& +\lambda\{28 \text { terms from all possible quadratic combinations among } \\
& \left.\hat{\alpha}_{i}, e_{i j}, \Omega_{i}, \psi_{i j k}, \theta_{i j}, \psi_{i}, \tau_{i},\left(\omega_{s}^{(0)}\right)_{i}\right\} \\
& +\lambda \epsilon_{1}\left\{4 \text { terms from time derivatives of } \hat{\alpha}_{i}, e_{i j}, \Omega_{i},\left(\omega_{s}^{(0)}\right)_{i}\right\}, \\
G_{i}= & G_{i}^{(0)}+\lambda G_{i}^{(1)} \\
= & \left\{B_{i j}^{1}\left[\hat{\alpha}_{j}-\lambda\left(U_{s}^{(1)}\right)_{j}\right]+{ }^{r} B_{i j k}^{1} e_{k j}+{ }^{r} A_{i j}^{1} \Omega_{j}+{ }^{r} B_{i j k l}^{1} \psi_{l k j}+{ }^{r} A_{i j k}^{1} \theta_{k j}\right. \\
& \left.+{ }^{r} B_{i j}^{2} \tau_{j}+{ }^{r} A_{i j}^{2}\left[\left(\omega_{s}^{(0)}\right)_{j}+\lambda\left(\omega_{s}^{(1)}\right)_{j}\right]\right\}+\lambda\{\ldots\}+\lambda \epsilon_{1}\{\ldots\} .
\end{aligned}
$$

Here, we have used $\hat{\alpha}_{i}$ to denote the combination $\alpha_{i}-\left(U_{s}^{(0)}\right)_{i}$. The ${ }^{t} A$ 's and ${ }^{r} A$ 's are second- and higher-rank time-dependent material tensors that depend upon the particle geometry, i.e. its shape and its orientation with respect to our non-rotating co-ordinate frame. By contrast, the ${ }^{t} B$ 's and ${ }^{r} B$ 's are second- and higher-rank time-dependent pseudo-tensors. This distinction between tensors and pseudo-tensors is necessary because vorticity and torque are pseudo-quantities. The time dependence of these coefficients reflects the changing geometry as the particle rotates, and is therefore not present when the particles are spheres. $\dagger$

So far, the shape of the particle has not been specified. Brunn (1977) considered the case of a transversely isotropic particle, i.e. a body of revolution with fore-aft symmetry. All odd-rank tensors and even-rank pseudo-tensors are then identically zero, while the rest depend on the orientation of the symmetry axis. The expressions for the force and torque to $O(1)$ and $O(\lambda)$ are then

$$
\begin{aligned}
F_{i}^{(0)}= & { }^{t} A_{i j}^{1} \hat{\alpha}_{j}+{ }^{t} A_{i j k l}^{1} \psi_{l k j}+{ }^{t} B_{i j k}^{1} \theta_{k j}+{ }^{t} A_{i j}^{2} \tau_{j}, \\
G_{i}^{(0)}= & { }^{r} B_{i j k}^{1} e_{k j}+{ }^{r} A_{i j}^{1} \Omega_{j}+{ }^{r} A_{i j}^{2}\left(\omega_{s}^{(0)}\right)_{j}, \\
F_{i}^{(1)}= & -{ }^{t} A_{i j}^{1}\left(U_{s}^{(1)}\right)_{j}+\left\{{ }^{t} B_{i j k}^{2} \hat{\alpha}_{k} \Omega_{j}+{ }^{t} B_{i j k}^{3} \hat{\alpha}_{k}\left(\omega_{s}^{(0)}\right)_{j}\right. \\
& +{ }^{t} B_{i j k}^{4} \Omega_{k} \tau_{j}+{ }^{t} B_{i j k}^{5}\left(\omega_{s}^{(0)}\right)_{k} \tau_{j}+{ }^{t} A_{i j k l}^{2} \hat{\alpha}_{l} e_{k j} \\
& +7 \text { more terms involving quadratic combinations of } e_{i j}, \psi_{i j k}, \\
& \theta_{i j}, \tau_{i}, \Omega_{i},\left(\omega_{s}^{(0)}\right)_{i} \text { with coefficients which are even-rank tensors or } \\
& \text { odd-rank pseudo-tensors }\}+\epsilon_{1}\left\{{ }^{t} A_{i j}^{2} \partial \hat{\alpha}_{j} / \partial t\right\},
\end{aligned}
$$

$\dagger$ Indeed, for analysis of non-spherical geometry it is more convenient to allow the co-ordinate frame to translate and rotate with the particle. Wo are mainly concerned in the present communication with spheres (equation (3.4) onwards) and so choose a non-rotating frame for our analysis. 


$$
\begin{aligned}
G_{i}^{(1)}= & { }^{r} A_{i j}^{2}\left(\omega_{s}^{(1)}\right)_{j}+\left\{r B_{i j k}^{2} \hat{\alpha}_{k} \hat{\alpha}_{j}+{ }^{r} B_{i j k}^{3} \hat{\alpha}_{k} \tau_{j}+{ }^{r} B_{i j k}^{4} \Omega_{k} \Omega_{j}\right. \\
& +13 \text { more terms involving quadratic combinations of } \hat{\alpha}_{i}, e_{i j}, \\
& \psi_{i j k}, \theta_{i j}, \tau_{i}, \Omega_{i},\left(\omega_{s}^{(0)}\right)_{i} \text { with coefficients which are even-rank } \\
& \text { tensors or odd-rank pseudo-tensors }\} \\
& +\epsilon_{1}\left\{{ }^{r} B_{i j k}^{5} \frac{\partial}{\partial t} e_{k j}+{ }^{r} A_{i j}^{3} \frac{\partial}{\partial t} \Omega_{j}+{ }^{r} A_{i j}^{4} \frac{\partial}{\partial t}\left(\omega_{s}^{(0)}\right)_{j}\right\} .
\end{aligned}
$$

\subsection{The rigid sphere}

To simplify further the calculations of this section, we consider the particular case of a neutrally buoyant rigid sphere in the absence of any externally applied torque. The non-zero material tensors and pseudo-tensors are then spherically isotropic and expressed in the most general form are

$$
\left.\begin{array}{c}
A_{i j}=a_{0} \delta_{i j}, \quad B_{i j k}=b_{0} \epsilon_{i j k}, \\
A_{i j k l}=a_{1} \delta_{i j} \delta_{k l}+a_{2} \delta_{i k} \delta_{j l}+a_{3} \delta_{i l} \delta_{j k} .
\end{array}\right\}
$$

Expressions for the higher-order tensor and pseudo-tensor coefficients follow in a similar manner but are excessively cumbersome, and will not be given here.

Equations (3.2) can now be further simplified. In particular

$$
{ }^{t} A_{i j k l}^{1} \psi_{l k j}=0, \quad{ }^{t} B_{i j k}^{1} \theta_{k j}={ }^{r} B_{i j k}^{1} e_{k j}=0,
$$

whereas for the other terms we may write

$$
\left.\begin{array}{c}
{ }^{t} A_{i j}^{1} \hat{\alpha}_{j}=c_{1}^{(0)} \hat{\alpha}_{i}, \quad{ }^{t} A_{i j}^{2} \tau_{j}=c_{2}^{(0)} \tau_{i}, \\
{ }^{r} A_{i j}^{1} \Omega_{j}=d_{1}^{(0)} \Omega_{i}, \quad{ }^{r} A_{i j}^{2}\left(\omega_{s}^{(0)}\right)_{j}=d_{2}^{(0)}\left(\omega_{s}^{(0)}\right)_{i} .
\end{array}\right\}
$$

Since the force and torque on the particle arc zero to the present level of approximation, we finally obtain

$$
\left(U_{s}^{(0)}\right)_{i}=\alpha_{i}+\frac{c_{2}^{(0)}}{c_{1}^{(0)}} \tau_{i}, \quad\left(\omega_{s}^{(0)}\right)_{i}=\left(-\frac{d_{1}^{(0)}}{d_{2}^{(0)}}\right) \Omega_{i}
$$

To simplify the $O(\lambda)$ expressions, we use $(2.12 b)$ for the time derivative of $\beta_{i j}\left[\hat{\alpha}_{i}\right.$ being constant with time by $(3.7 a)$ ], relationships analogous to $(3.5)$ and (3.6), and also $(3.7 b)$. This leads to

$$
\begin{aligned}
& \left(U_{\mathrm{g}}^{(1)}\right)_{i}=\left(c_{1}^{(0)}\right)^{-1}\left\{c_{1}^{(1)} \hat{\alpha}_{m} e_{m i}+c_{2}^{(1)} \epsilon_{i m n} \hat{\alpha}_{m} \Omega_{n}+c_{3}^{(1)} e_{n m n} \psi_{m n i}+c_{4}^{(1)} \epsilon_{i m n} e_{m l} \theta_{l n}+c_{5}^{(1)} e_{i m} \tau_{m}\right. \\
& \left.+c_{\mathrm{b}}^{(1)} \Omega_{m} \theta_{m i}+c_{7}^{(1)} \varepsilon_{i m n} \Omega_{n} \tau_{m}\right\}, \\
& \left(\omega_{s}^{(1)}\right)_{i}=-\left(d_{2}^{(0)}\right)^{-1}\left\{d_{1}^{(1)} \hat{\alpha}_{m} \theta_{m i}+d_{2}^{(1)} e_{i m} \Omega_{m}+d_{3}^{(1)} \psi_{i m n} \theta_{n m}\right. \\
& \left.+d_{4}^{(1)} \theta_{i m} \tau_{m}+d_{5}^{(1)}\left(U_{s}^{(0)}\right)_{m} \theta_{m i}+d_{6}^{(1)} \epsilon_{i m n}\left(U_{s}^{(0)}\right)_{m} \tau_{n}\right\}
\end{aligned}
$$

Equations $(3.7 a, b)$ and $(3.8 a, b)$ are the main results of this section. $\left(U_{s}^{(0)}\right)_{i}$ and $\left(\omega_{s}^{(0)}\right)_{i}$ are the translational and angular velocities of a sphere in an unbounded Newtonian fluid. The scalars $c_{1}^{(0)}, c_{2}^{(0)}, d_{1}^{(0)}$ and $d_{2}^{(0)}$ in (3.6) are

$$
c_{1}^{(0)}=6 \pi, \quad c_{2}^{(0)}=2 \pi, \quad d_{1}^{(0)}=-d_{2}^{(0)}=8 \pi .
$$

Hence, as expected, the angular velocity is equal to the local undisturbed vorticity, whereas the translational velocity shows the anticipated dependence on the undisturbed fluid velocity $\alpha_{i}$ plus an additional term related to the existence of a gradient 
in the rate of strain. These well-known results are of course completely consistent with, and derivable from, Faxén's laws (Brenner 1964).

The $O(\lambda)$ non-Newtonian contributions to the translational and angular velocities of the sphere are given by (3.8b). To make these equations useful, we need of course to obtain $c_{i}^{(1)}$ and $d_{i}^{(1)}$. To this end, we may simply follow the reciprocal theorem outlined by Ho \& Leal (1976). However, since they have already performed the migration calculations for a two-dimensional unidirectional flow, we need only a careful comparison of the general equation (3.8a) with their specific results to determine completely the coefficients $c_{i}^{(1)}$. The flow parameters for the two-dimensional case are

$$
\alpha_{i}=\delta_{1 i} \alpha, \quad \beta_{i j}=\delta_{1 i} \delta_{3 j} \beta, \quad \gamma_{i j k}=\delta_{1 i} \delta_{3 j} \delta_{3 k} \gamma
$$

For the purpose of comparing (3.8a) term by term with the migration-velocity expression of Ho \& Leal (1976), it is important to distinguish the separate contributions of $\psi_{i j k}, \theta_{i j}$ and $\tau_{i}$ in the original two-dimensional calculation. After some work, we finally obtain

$$
\begin{gathered}
c_{1}^{(1)}=6 \pi\left(1+2 \epsilon_{1}\right), \quad c_{2}^{(1)}=6 \pi \epsilon_{1}, \quad c_{3}^{(1)}=\frac{5}{3} \pi\left(5+13 \epsilon_{1}\right), \\
c_{4}^{(1)}=\frac{2}{9} \pi\left(1+11 \epsilon_{1}\right), \quad c_{5}^{(1)}=2 \pi\left(2+5 \epsilon_{1}\right), \quad c_{6}^{(1)}=0, \quad c_{7}^{(1)}=2 \pi \epsilon_{1} .
\end{gathered}
$$

To illustrate further the physical significance of the $O(\lambda)$ translational-migration expression, we substitute (3.7a), (3.9) and (3.11) into (3.8a) to obtain

$$
\left(U_{8}^{(1)}\right)_{i}=\frac{5}{18}\left(5+13 \epsilon_{1}\right) e_{n m} \psi_{m n i}+\frac{1}{27}\left(1+11 \epsilon_{1}\right) \epsilon_{i m n} e_{m l} \theta_{l n}+\frac{1}{3}\left(1+3 \epsilon_{1}\right) e_{i m} \tau_{m}
$$

Qualitatively, we see for the case of a neutrally buoyant rigid sphere that $\left(U_{s}^{(1)}\right)_{i}$ is generated only from the interaction between the pure straining part of the linear contribution to $V_{i}$ and either a strain-rate gradient or a vorticity gradient. The constant vorticity $\Omega_{i}$ has no effect. The accepted value for $\epsilon_{1}$ is (Ho \& Leal 1976)

$$
-0.6<\epsilon_{1}<-0.5
$$

and therefore the coefficients in (3.12) are all negative. In general, it is not possible to predict the direction of motion without specific knowledge of the flow parameters $e_{i j}, \psi_{i j k}, \theta_{i j}$ and $\tau_{i}$. However, we observe, after some straightforward algebra, that the absolute shear rate for the bulk flow is given by

$$
e_{i m} e_{m i}+\left[4 e_{n m} \psi_{m n i}+\frac{2}{3} e_{i m n} e_{m l} \theta_{l n}+\frac{6}{5} e_{i m} \tau_{m}\right] x_{i}+\ldots
$$

By inspection, we see then that in general each separate term in (3.12) tends to induce $O(\lambda)$ migration in the direction of decreasing absolute shear rate, in agreement with the conclusions of Ho \& Leal (1976).

The above results $(3.8 a)$ and (3.12) are particularly important when $\left(U_{8}^{(1)}\right)_{i}$ represents the first non-zero (though $O(\lambda)$ ) contribution to the motion of the sphere in the lateral direction. This happens when, in the Newtonian limit, the sphere translates only in the direction of the bulk translational velocity (i.e. $\epsilon_{i j k} \alpha_{k} \tau_{j}=0$ ), as is always the case when the bulk flow itself is unidirectional. If it does not, then we see from $(3.7 a)$ that the first lateral motion still occurs at $O(\lambda)$ if $\left(U_{s}^{(1)}\right)_{i}$ has a component orthogonal to the $\alpha_{i}, \tau_{i}$ plane (i.e. $\epsilon_{i j k} \alpha_{k} \tau_{j}\left(U_{s}^{(1)}\right)_{i} \neq 0$ ). Meanwhile the components of $\left(U_{s}^{(1)}\right)_{i}$ in the $\alpha_{i}, \tau_{i}$ plane represent only small non-Newtonian corrections to the translational motion of the particle. 


\subsection{The spherical drop}

Let us now turn to the interesting case where the particle is a non-Newtonian drop with zero-shear viscosity $\tilde{\mu}_{0}$. To non-dimensionalize the equations of motion inside the drop, we use a characteristic pressure $\tilde{\mu}_{0} G$ with all other characteristic quantities as defined in $\S 2$. The equations become

$$
\partial \tilde{S}_{i j} / \partial x_{j}=0, \quad \partial \tilde{D}_{i} / \partial x_{i}=0
$$

where

$$
\tilde{S}_{i j}=-\tilde{P} \delta_{i j}+\tilde{D}_{(1) i j}+\tilde{\lambda} \tilde{D}_{(1) i k} \tilde{D}_{(1) k j}+\tilde{\lambda} \tilde{\epsilon}_{1} \tilde{D}_{(2) i j}
$$

The boundary conditions in this case are more complicated. In particular it will be futile to define an angular velocity of the drop analogous to (2.8). On the surface of the neutrally buoyant drop, the boundary conditions are

$$
\begin{gathered}
U_{i}=\tilde{U}_{i} \quad \text { (matching velocity), } \\
U_{i} n_{i}=\tilde{U}_{i} n_{i}=0 \quad \text { (kinematic condition), } \\
S_{i j} n_{j}=\kappa \widetilde{S}_{i j} n_{j}+\frac{1}{\delta}\left(\frac{1}{R_{1}}+\frac{1}{R_{2}}\right) n_{i} \quad \text { (matching stress). }
\end{gathered}
$$

Here $\kappa=\tilde{\mu}_{0} / \mu_{0}, \delta=a \mu_{0} G / \sigma$, where $\sigma$ is the interfacial tension, and $R_{1}$ and $R_{2}$ are the principal radii of curvature.

In contrast to a rigid particle, it is not possible in the case of a drop to obtain general expressions for the particle motion simply by force and torque considerations alone. Since no angular velocity can be defined, a torque expression will be useless. We note, however, that the condition of no torque on the particle is already implied by $(3.17 c)$.

The translational migration velocity can still be obtained. By considering the force alone, we get, for the case of an undeformed spherical drop (i.e. $\delta=0$ ),

$$
\begin{gathered}
\left(\hat{U}_{s}^{(0)}\right)_{i}=\alpha_{i}+\left(\hat{c}_{2}^{(0)}\left(\hat{c}_{1}^{(0)}\right) \tau_{i},\right. \\
\left(\hat{U}_{s}^{(1)}\right)_{i}=\left(\hat{c}_{1}^{(0)}\right)^{-1}\left\{\hat{c}_{1}^{(1)}\left[\alpha_{m}-\left(\hat{U}_{s}^{(0)}\right)_{m}\right] e_{m i}+\hat{c}_{2}^{(1)} \epsilon_{i m n}\left[\alpha_{m}-\left(\hat{U}_{s}^{(0)}\right)_{m}\right] \Omega_{n}\right. \\
\left.+\hat{c}_{3}^{(1)} e_{n m} \psi_{m n i}+\hat{c}_{4}^{(1)} \epsilon_{i m n} e_{m l} \theta_{l n}+\hat{c}_{5}^{(1)} e_{i m} \tau_{m}+\hat{c}_{6}^{(1)} \Omega_{m} \theta_{m i}+\hat{c}_{7}^{(1)} \epsilon_{i m n} \Omega_{n} \tau_{m}\right\} .
\end{gathered}
$$

Again, $\hat{c}_{\mathbf{1}}^{(0)}$ and $\hat{c}_{2}^{(0)}$ in (3.18) are well known, and given by

$$
\hat{c}_{1}^{(0)}=2 \pi\left(\frac{2+3 \kappa}{1+\kappa}\right), \quad \hat{c}_{2}^{(0)}=2 \pi\left(\frac{\kappa}{1+\kappa}\right) .
$$

As for the $O(\lambda)$ expression (3.19), we get, again by comparison with a detailed reciprocal-theorem calculation (Chan \& Leal 1977),

$$
\begin{aligned}
& \hat{c}_{1}^{(1)}=\frac{2 \pi}{5(1+\kappa)^{3}}\left[\left(12+32 \kappa+40 \kappa^{2}+15 \kappa^{3}\right)+\epsilon_{1}\left(22+61 \kappa+80 \kappa^{2}+30 \kappa^{3}\right)\right] \\
& +\eta \frac{12 \pi}{5(1+\kappa)^{3}}\left[1+2 \tilde{\epsilon}_{1}\right], \\
& \begin{aligned}
\hat{c}_{2}^{(1)}=\frac{2 \pi}{(1+\kappa)^{2}} \epsilon_{1}\left(2+4 \kappa+3 \kappa^{2}\right)+\eta \frac{2 \pi}{(1+\kappa)^{2}} \tilde{\epsilon}_{1}, \\
\hat{c}_{3(}{ }^{1)}=\frac{\pi}{63(1+\kappa)^{3}}\left[\left(256+816 \kappa+1238 \kappa^{2}+525 \kappa^{3}\right)\right. \\
\left.\quad+\epsilon_{1}\left(592+2112 \kappa+3254 \kappa^{2}+1365 \kappa^{3}\right)\right]+\eta \frac{8 \pi}{21(1+\kappa)^{3}}\left[8+17 \tilde{\epsilon}_{1}\right],
\end{aligned}
\end{aligned}
$$


$\hat{c}_{4}^{(1)}=\frac{2 \pi}{27(4+\kappa)(1+\kappa)^{2}}\left[\left(16+60 \kappa+11 \kappa^{2}+3 \kappa^{3}\right)+\epsilon_{1}(64+204 \kappa\right.$

$$
\left.\left.+173 \kappa^{2}+33 \kappa^{3}\right)\right]+\eta \frac{4 \pi}{9(4+\kappa)(1+\kappa)^{2}}\left[-5+\tilde{\epsilon}_{1}\right]
$$

$\hat{c}_{5}^{(1)}=\frac{2 \pi}{5(1+\kappa)^{3}}\left[\left(4+11 \kappa+22 \kappa^{2}+10 \kappa^{3}\right)+\epsilon_{1}\left(8+28 \kappa+56 \kappa^{2}+25 \kappa^{3}\right)\right]+\eta \frac{12 \pi}{5(1+\kappa)^{3}}\left[1+2 \tilde{\epsilon}_{1}\right]$,

$\hat{c}_{6}^{(1)}=0, \quad \hat{c}_{7}^{(1)}=\frac{2 \pi}{(1+\kappa)^{2}} \epsilon_{1} \kappa^{2}+\eta \frac{2 \pi}{(1+\kappa)^{2}} \tilde{\epsilon}_{1}$,

where $\eta=(\check{\lambda} / \lambda) \kappa$. These results of course agree with those for a rigid sphere when $\kappa$ approaches infinity.

If we now substitute (3.18), (3.20) and (3.21) into (3.19), an expression analogous to (3.12) can be obtained. For the sake of brevity, we shall omit the cumbersome expression which results. However, we note, in contrast to the previous case of a rigid sphere, that constant vorticity will also contribute to the migration velocity of a neutrally buoyant drop. It is also obvious, since both fluids can make 'independent' contributions to the drop motion at $O(\lambda)$ [ef. (3.21)], that migration will still occur even if only the drop fluid is non-Newtonian.

\section{Discussion}

In the preceding section, we have derived expressions from which the first nonNewtonian contributions to the motion of spherical particles or drops can be calculated exactly for a general quadratic flow of a second-order fluid. An approximate scheme which might appear to be an attractive alternative for unidirectional shear flows is simply to assume that the undisturbed flow is locally two-dimensional so that the results of Ho \& Leal (1976) can be adopted directly. In this section we compare the exact and approximate predictions for the case of a rigid sphere in a pressure-driven flow through a straight tube of elliptical cross-section.

A cross-sectional view of the configuration which we consider is shown in figure 1. We assume the unidirectional flow to be in the $X_{1}^{\prime}$ direction, so that the undisturbed velocity profile is given by $\dagger$

$$
V_{i}^{\prime}=V_{\max }\left(1-\frac{X_{2}^{\prime 2}}{A_{0}^{2}}-\frac{X_{3}^{\prime 2}}{B_{0}^{2}}\right) \delta_{1 i}
$$

where $A_{0}$ and $B_{0}$ are the major and minor semi-axes respectively. We let $\left(\bar{x}_{2}, \bar{x}_{3}\right)$ be the components of the position vector for any material point in a co-ordinate system nondimensionalized with the sphere radius $a$ and fixed at the sphere centre. The sphere itself is at a radial distance $D$ from the tube axis. Hence

$$
X_{2}^{\prime}=D \sin \theta+a \bar{x}_{2}, \quad X_{3}^{\prime}=D \cos \theta+a \bar{x}_{3} .
$$

By straightforward substitution into (4.1), we may express the undisturbed velocity $V_{i}$ relative to the sphere as

$$
V_{i}=V_{\max }\left\{\left[1-s^{2}\left(p^{2} \sin ^{2} \theta+\cos ^{2} \theta\right)\right]-2 s \zeta\left(p^{2} \sin \theta \bar{x}_{2}+\cos \theta \bar{x}_{3}\right)-\zeta^{2}\left(p^{2} \bar{x}_{2}^{2}+\bar{x}_{3}^{2}\right)\right\} \delta_{1 i}-\left(U_{s}\right)_{i}
$$

$\dagger$ This approximation can be shown to be accurate to $O\left(\lambda^{4}\right)$ in the retarded-motion, $n$ th-order fluid expansion, cf. Langlois \& Rivlin (1963). 


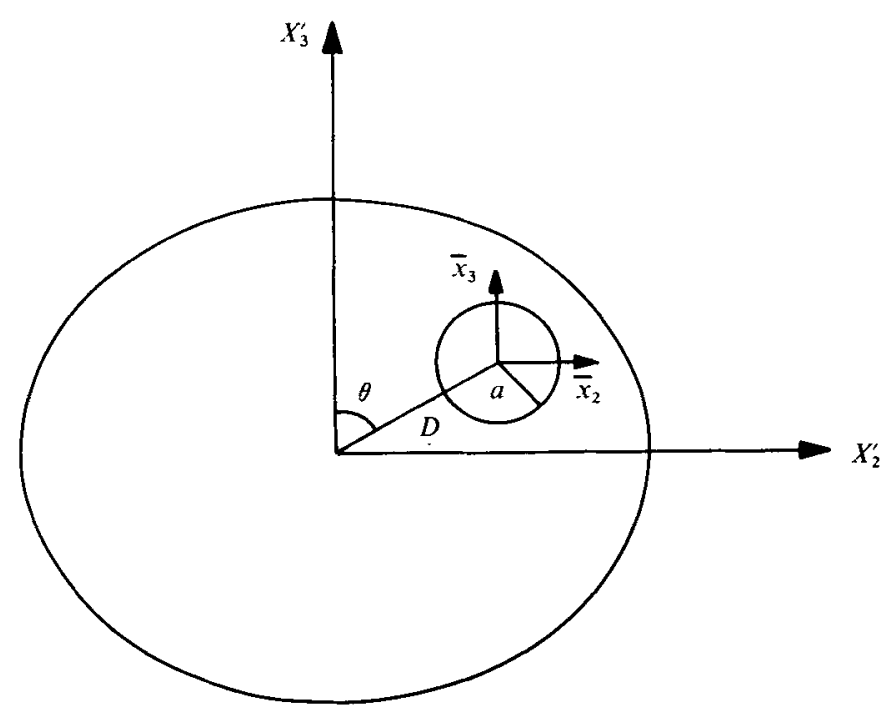

Figure 1. A cross-sectional view of a sphere in an elliptical tube.

where $p=B_{0} / A_{0}, s=D / B_{0}$ and $\zeta=a / B_{0}$. The parameter $p$ is zero for the limiting case of two parallel plates and unity for a circular tube, whereas $s$ is $O(1)$. The basic theory neglects wall effects and hence implicitly assumes $\zeta$ to be small.

To consider the migration velocity of the sphere, it is most convenient to use co-ordinates $\left(x_{2}, x_{3}\right)$ defined by the orthogonal transformation

$$
\left.\begin{array}{l}
\bar{x}_{2}=\left(p^{4} \sin ^{2} \theta+\cos ^{2} \theta\right)^{-\frac{1}{2}}\left(\cos \theta x_{2}+p^{2} \sin \theta x_{3}\right), \\
\bar{x}_{3}=\left(p^{4} \sin ^{2} \theta+\cos ^{2} \theta\right)^{-\frac{1}{2}}\left(-p^{2} \sin \theta x_{2}+\cos \theta x_{3}\right) .
\end{array}\right\}
$$

The undisturbed velocity may then be expressed as

where

$$
V_{i}=\left[\alpha+\beta x_{3}+\gamma\left(x_{3}^{2}+\phi_{1} x_{2} x_{3}+\phi_{2} x_{2}^{2}\right)\right] \delta_{1 i}-\left(U_{s}\right)_{i}
$$

$$
\left.\begin{array}{c}
\alpha=V_{\max }\left[1-s^{2}\left(p^{2} \sin ^{2} \theta+\cos ^{2} \theta\right)\right], \quad \beta=V_{\max }\left[-2 s \zeta\left(p^{4} \sin ^{2} \theta+\cos ^{2} \theta\right)^{\frac{1}{2}}\right] \\
\gamma=V_{\max }\left[-\frac{\zeta^{2}\left(p^{6} \sin ^{2} \theta+\cos ^{2} \theta\right)}{p^{4} \sin ^{2} \theta+\cos ^{2} \theta}\right]
\end{array}\right\}
$$

and

$$
\phi_{1}=\frac{2 p^{2}\left(p^{2}-1\right) \sin \theta \cos \theta}{p^{6} \sin ^{2} \theta+\cos ^{2} \theta}, \quad \phi_{2}=\frac{p^{2}\left(p^{2} \sin ^{2} \theta+\cos ^{2} \theta\right)}{p^{6} \sin ^{2} \theta+\cos ^{2} \theta} .
$$

Equation (4.5) is in the most advantageous form for our present analysis. The 'crossstream' direction, in which uniform shearing occurs, is now denoted by $x_{3}$ and $\phi_{1}$ and $\phi_{2}$ are parameters which reduce to zero for the limiting case of two parallel plates.

The approximate procedure which we outlined at the beginning of this section is to assume the undisturbed flow to be locally two-dimensional so that the results of Ho \& Leal (1976) can be adopted directly. The most natural implementation of this scheme in the present case is to take the $x_{1}, x_{3}$ plane as the (local) plane of shear, i.e. to neglect completely the $\phi_{1}$ and $\phi_{2}$ contributions to the shear gradient. The analysis of Ho \& Leal (1976) then gives

$$
\left(U_{s}^{(1)}\right)_{i}=\beta \gamma\left[\frac{5}{9}\left(1+3 \epsilon_{1}\right)\right] \delta_{3 i}
$$


This is to be compared with the exact result for the migration velocity, which may be calculated using (3.12) in conjunction with (4.5)-(4.7). After some algebra, we obtain

$$
\left(U_{s}^{(1)}\right)_{i}=\beta \gamma\left\{\left[\frac{5}{9}\left(1+3 \epsilon_{1}\right)+\frac{\phi_{2}}{18}\left(1+\epsilon_{1}\right)\right] \delta_{3 i}+\frac{\phi_{1}}{36}\left(9+29 \epsilon_{1}\right) \delta_{2 i}\right\}
$$

The above expresson, with parameters $\beta$ and $\gamma$, clearly reduces in the limit as $p$ approaches zero to that of Ho \& Leal (1976) for the case of two parallel plates. Furthermore, comparison of (4.8) and (4.9) shows, in general, that the attempt to adapt directly the two-dimensional results of Ho \& Leal to the elliptical-tube problem [i.e. use (4.8) as an approximation] leads to a predicted migration velocity which not only has an error in magnitude, but is also in a slightly incorrect direction [cf. the $\phi_{1}$ term in (4.9)]. This difference in direction is zero for the particular case of a circular tube, but is significant when the parameter $p$ is about 0.7 . For the circular tube, we see from (4.4) that $x_{3}$ corresponds exactly to the radial direction and hence the sphere migrates in a straight line towards the tube centre. The difference in magnitude between (4.8) and (4.9) is then less than $10 \%$, assuming $\varepsilon_{1}=-0.55$ [cf. (3.13)]. For the elliptical tube, however, the sphere usually describes a curved trajectory in the $x_{2}^{\prime}, x_{3}^{\prime}$ plane which, at any instant, depends on its radial distance $D$ and orientation relative to the tube centre. (On the major and minor axes, the trajectories will be straight lines.) Intuitively, it is reasonable to assume that the sphere will move towards the centre, but we shall not attempt to provide a rigorous proof here.

The authors thank Dr H. Brenner for two very interesting seminars and subsequent discussions, which inspired this paper. This work was supported by NSF Grant ENG74-17590.

\section{REFERENCES}

BrenNer, H. 1964 Chem. Engng Sci. 19, 703.

BronN, P. 1976 Rheol. Acta 15, 163.

BrunN, P. 1977 J. Fluid Mech. 82, 529.

Caswell, B. \& Schwarz, W. H. 1962 J. Fluid Mech. 13, 417.

Chan, P. C.-H. \& Leat, L. G. 1977 The motion of a deformable drop in a second-order fluid. In preparation.

Coope, J. A. R., Snider, R. F. \& McCourt, F. R. 1965 J. Chem. Phys. 43, 2269.

Fredrickson, A. G. 1964 Principles and Applications of Rheology. Prentice-Hall.

HINCH, E. J. 1972 J. Fluid Mech. 54, 423.

Ho, B. P. \& LeaL, L. G. 1976 J. Fluid Mech. 76, 783.

Langlots, W. E. \& Rrvitn, R. S. 1963 Rend. Mat. Appl. Univ. Roma, Inst. Naz. Alta Mat. 22, 169. 